\title{
Silver nanoparticles (AgNPs): as nanopesticides and nanofertilizers
}

\begin{abstract}
Nanotechnology is evolving as an important field of sciences due to its promising role in the agricultural sector. Nanotechnology is being increasingly used to improve crop yield and reducing the harmful agents used in crop protection. Recently silver nanoparticles have gained utmost attention due its uses in various areas of human interest whether it be agriculture, industry, medicine and human health. They are the most popular metallic nanoparticles and its various antimicrobial and antioxidant as well as anticancer properties have been reported. This mini review aims to present and discuss development of cost effective techniques for the synthesis and recovery of silver nanoparticles that have agricultural importance. Many of its applications in plant disease control and other aspects such as their role as nanopesticides or nanofertilizers have been discussed.
\end{abstract}

Keywords: Silver Nanoparticles, nanopesticides, agrochemicals, nanoencapsulation
Volume 4 Issue I - 2019

\author{
Rythem Anand, Madhulika Bhagat \\ School of Biotechnology, University of Jammu, Jammu, J\&K- \\ 180006, India
}

\author{
Correspondence: Madhulika Bhagat, School of Biotechnology, \\ University of Jammu, Jammu, J\&K-180006, India, \\ Email madhulikasbt@gmail.com
}

Received: November 23, 2018 | Published: February 18, 2019

\section{Introduction}

Nanotechnology is a new and promising area of research having particles with improved properties of size and morphology than the bulk substances. Nanoparticles have their size in the range of 1-100nm which increases the surface area of the particles and this increase in surface area thus enhances the biological activity of the nanoparticles. Although there are many measures involved in the synthesis of the nanoparticles, there is an vital need to develop a cost effective, non toxic and eco-friendly method corresponding to high yields. These nanomaterials are economical and therefore act as bio-nano factories which provide an additional benefit of being used in lesser quantities. These materials have role in enhancement of agricultural productivity involving nanoporous materials for slow release and efficient dosage of pesticides and fertilizers leading to pest management. Nanoparticles have distinct properties whether it be physical, chemical and biological as compared to larger particle of bulk material. It has been well established that the metallic nanoparticles have unique catalytic activity in removal of the contaminants both organic as well as inorganic. ${ }^{1}$ The application of nanotechnological methods in plant pathology is still in the early stages for various agricultural purpose like development of the nanoformulations of pesticides, fungicides, herbicides, fertilizers, insecticides etc. Efficient nano-delivery systems help in minimizing the highly toxic fungicides and pesticides used in the agricultural sector. Similarly, nanoparticles help in gene transfer which acts as ideal tool to grow crops and plants resistant to pathogens and pests. ${ }^{2}$ Nanomaterials can be used as a carrier for the agrochemicals or used as biopesticides or biofertilizers.

\section{Green Synthesis of silver nanoparticles}

Silver has been used since time immemorial because of its various inhibitory properties against various microorganisms. Silver nanoparticles have been gaining attention of the researchers because of its distinct properties in terms of optical, antimicrobial and antioxidant ones. Nanoparticles can be prepared in several ways such as gamma irradiation, chemical reduction, photochemical methods, and other biological methods ${ }^{3}$ and various nanoparticles are produced by green synthesis using biological substances leading to high yield that are environment friendly and cost effective. ${ }^{4,5}$

Silver nanoparticles (AgNPs) are synthesized using a reduction of aqueous $\mathrm{Ag}+$ ion by various biological agents whether it be microbes of the plant extracts. These reactions take place at ambient temperature that is the room temperature and can further be monitored by ultraviolet-visible spectroscopy and its surface morphology studied by transmission electron microscopy , scanning electron microscopy. The elemental composition of the particles was studied by X-ray diffraction. ${ }^{5,6}$ Thus, silver nanoparticles came out with diverse applications in the agricultural sector as well as medical and industrial sectores. ${ }^{7}$

\section{Nanoparticles in agro systems}

Nanoparticles have proved to be efficient agrochemical agents in order to improve the crop productivity and reducing the pests. They have boosted the agricultural application by increasing the nutrient uptake by plants and also inhibiting the pathogens infecting the plants. Nanoparticles act as 'magic bullets' serving as herbicides, pesticides and fertilizers etc. Nanoparticles penetrate the cuticle and tissues of the plants very well thus allowing their slow and effective release to the target area.

\section{Silver Nanoparticles as nanopesticides}

Pests are increasing threat to the agricultural sector leading to the decrease in crop yield and thereby reducing the quality of the crops too. Moreover, the synthetic pesticides applied to the soil or plants have negative impacts on the environment. ${ }^{8}$ The pest management can be done by involving nanotechnology into it. Nano-silver acts as an effective agent in pest management that is non toxic, safe and an improved tool to fight pests and moreover the silver nanoparticle based pesticide provides high dose of pesticides to the target plants. ${ }^{9}$ In the green method of silver nanoparticles preparation, the biological agents used for their synthesis can be microbes or plants and the flavinoids present in the plants proves to be toxic to the plants. Thus the pesticidal activity of the silver nanoparticles can help a lot in the 
pest control. ${ }^{10}$ Moreover antifungal activity of the silver nanoparticles have also been reported and thus they can be used in treating the fungal pathogens of the plants. ${ }^{11}$ Sap-lam et al., ${ }^{12}$ reported the potential of UV-irradiated induced silver nanoparticles in the biocontrol of pests such as mosquito larvae.

\section{Silver nanoparticles as nanofertilizers}

Nowadays, large amounts of commercial fertilizers are used in the form of urea and nitrate etc but they have toxic effects on the plants and the beneficial microflora. They cause pollution too. Nano materials can help in this area as the they allow slow release of the agrochemical due to high surface area of the particles than the conventional ones. Silver nanoparticles have many unique properties and silver being a nutrient for the plants can be used to increase the nutrient uptake efficiency of the plants. The nano-silver provides an improved uptake of nutrients from the soil than the bulk one. Therefore it can have applications in the crop protection as well as crop protection. They can be used as nanofertilizers. It has been reported that silver nanoparticles could be used to enhance seed germination potential in many plants. ${ }^{4}$ Nanofertilizers help in the slow and effective release of the nutrients, thereby preventing the nutrient losses. Absorption of nutrients by the plants from soil can be maximized using nanofertilizer. ${ }^{13-18}$

\section{Conclusion}

Nanotechnology has provided solutions to many agriculturerelated problems like insect pest management as well as nutrient uptake efficiency etc. Nanomaterials in different forms can be used for efficient pest management and crop production. Biosynthesized silver nanoparticles (AgNPs) have proved its application in performing two major tasks of plant growth enhancement and plant disease management. Therefore, it can also be concluded that silver nanoparticles proves to be an efficient tool in providing green and eco-friendly alternatives. They have antimicrobial activity against the various plant pathogens and moreover help the plants in absorbing important nutrients from the soil.

\section{Acknowledgments}

None.

\section{Conflict of interest}

Author declares their no conflict of interest.

\section{References}

1. Manimegalai G, Shanthakumar S, Sharma C. Silver Nanpraticles: synthesis and application in mineralization of pesticides using membrane support. Int Nano letter. 2014;4:105.

2. Wang A, Wang Y, Sun C, et al. Fabrication, Characterization, and Biological Activity of Avermectin Nano-delivery Systems with Different Particle Sizes. Nanoscale Res Lett. 2018;13(1):2.

3. Remya VR, Abitha VK, Rajput PS, et al. Silver nanoparticles green synthesis: A mini review. Chemistry International. 2017;3(2):165.

4. Duhan JS, Kumar R, Kumar N, et al. Nanotechnology: The new perspective in precision agriculture. Biotechnol Rep. 2017;11-23.

5. Li G, He D, Qian Y, et al. Fungus-Mediated Green Synthesis of Silver Nanoparticles Using Aspergillus terreus. Int J Mol Sci. 2012;13(1):466476.

6. Shao $\mathrm{Y}, \mathrm{Wu} \mathrm{C}, \mathrm{Wu} \mathrm{T}$, et al. Green synthesis of sodium alginatesilver nanoparticles and their antibacterial activity. Int $J$ Biol Macromol. 2018;111:1281-1292.

7. Rai M, Ingle A. Role of nanotechnology in agriculture with special reference to management of insect pests. Appl Microbiol Biotechnol. 2012;94(2):287-293

8. Francesco Lai, Sylvia A Wissing Rainer H, et al. Artemisia arborescens L Essential Oil-Loaded Solid Lipid Nanoparticles for Potential Agricultural Application: Preparation and Characterization. AAPS Pharm Sci Tech. 2006;7(1):E10.

9. Ragaei M, Hasaan Sabry A. Nanotechnology for insect pest control. International Journal of Science, Enironment and Technology. 2014;3(2):528-545.

10. Zahir AA, Bagavan A, Kamaraj C, et al. Efficacy of plant mediated synthesized silver nanoparticles against Sitophilus oryzae. $J$ Biopest. 2012;5(Supplementary):95-102.

11. Kim SW, Jung JH, Lamsal K, et al. Antifungal effects of silver nanoparticles (AgNPs) against various plant pathogenic fungi. Microbiology. 2012;40(1):53-58.

12. Sap-lam N, Homklinchan C, Larpudomlert R, et al. UV irradiation induced silver nanoparticles as mosquito larvicides. Journal of Applied Sciences. 2010;10(23):3132-3136. 